\title{
Computing Complexity of Symmetric Quadratic Neural Networks ${ }^{1}$
}

Eric Goles

Universidad de Chile, Facultad de Ciencias
Martín Matamala**

Laboratoire de l'Informatique du Paralléllisme

Físicas y Matemá ticas, Departamento de Ingeniería Ecole Normale Supérieure de Lyon

Matemá tica. Casilla 170-correo 3 Santiago, Chile 46, Allée d'Italie, 69364 Lyon Cedex 07, France

\section{Extended Abstract}

A neural network of order $p \in \mathbb{N}$ (p-PNN in the sequel) $[1,4]$ is defined as a set of $n$ neurons taking values in $\{0,1\}$ and interconnected by a tuple $\left(A^{(p)}, A^{(p-1)}, \ldots, A^{(0)}\right)$ where $A^{(p)}=\left(a_{i_{0}, i_{1}, \ldots, i_{p}}^{(p)} \in\right.$ $\mathbb{R}^{(p+1) n}, \ldots, A^{(0)}=\left(a_{i_{0}}^{(0)}\right) \in \mathbb{R}^{n}$. The update function is as follows: For $1 \leq i_{0} \leq n$

$y_{i_{0}}=\mathbb{1}\left(\frac{1}{p !} \sum_{i_{1}, \ldots, i_{p}=1}^{n} a_{i_{0} i_{1} \ldots i_{p}}^{(p)} x_{i_{1}} \cdots x_{i_{p}}+\ldots+\frac{1}{2} \sum_{i_{1}, i_{2}=1}^{n} a_{i_{0} i_{1} i_{2}}^{(2)} x_{i_{1}} x_{i_{2}}+\sum_{i_{1}=1}^{n} a_{i_{0} i_{1}}^{(1)} x_{i_{1}}+a_{i_{0}}^{(0)}\right)$

where $\mathbb{1}(u)=1$ iff $u \geq 0$ ( 0 otherwise).

The most usual dynamics for the previous network are the sequential and the parallel iteration. In the former case all cells are updated one by one in a prescribed order. The parallel iteration consists to update all the cells synchronously. We introduce also some hypothesis on the arrays $\left(A^{(p)}, \ldots . A^{(0)}\right)$; Symmetry: $\forall 1 \leq s \leq p$, for any permutation, $\sigma$, of the indices $: i_{0}, \ldots, i_{s} \in\{1, \ldots, n\}$ $a_{i_{0} i_{1} \ldots i,}^{(s)}=a_{\sigma\left(i_{0}\right) \sigma\left(i_{1}\right) \ldots \sigma\left(i_{0}\right)}^{(s)}$ and Non-negativity: $\forall 1 \leq s \leq p$, if at least two indices have the same value; i.e. when $\left|\left\{i_{0}, i_{1}, \ldots, i_{s}\right\}\right| \leq s$, then $a_{i_{0}, \ldots, i_{s}}^{(s)} \geq 0$. Under these hypothesis one gets, for $p=1$, the usual Hopfield model [3] of a linear symmetric neural networks: $y_{i}=\mathbb{1}\left(\sum_{j=1}^{n} a_{i j} x_{j}-\theta_{i}\right)$, where $A=\left(a_{i j}\right)$ is symmetric and $\operatorname{diag}(A) \geq 0$.

Here, we present a simulation of arbitrary (non necessarily symmetric) linear neural networks by symmetric neural networks with quadratic $(q=2)$ arguments (2-PSNN). In order to make the simulation, we introduce a special unit: the $\Delta$-unit which consists of two neurons connected by a quadratic weight. This unit can orientate the information and then it can simulate the interaction $a_{i j}$ between two neurons $i$ and $j$ in the linear network.

Our general result is given by the following theorem whose proof can be seen in [2].

Theorem . Let $(A, \theta)$ be a linear neural network of size $n$, then there exists a 2-PSNN with $2(n+1)$ $\Delta$-units and a morphism $\varphi:\{0,1\}^{n} \rightarrow\{0,1\}^{2(n+1)}$ such that: $\forall x \in\{0,1\}^{n} \quad \varphi(F(x))=G^{2}(\varphi(x))$, where $F$ and $G$ are the global update functions of the linear and quadratic network respectively.

\section{References}

[1] Chen, H.H., Lee, Y.C., Maxwell, T., Sun, G.Z., Lee, H.Y., \& Giles, C.L., (1986) 'High order correlation model for associative memory'. AIP Conference Proceeding, in J.S. Denker (Eds), Neural networks for computing, pp 86-92.

[2] Goles, E., Matamala, M. (1992) 'Dynamical and Complexity Results for High Order Neural Networks' Research Report, Dep. Ingeniería Matemática, Facultad Ciencias Físicas y Matemáticas, Univ. de Chile.

[3] Hopfield, J.J. (1982), 'Neural Networks and Physical Systems with Emergent Collective Computational Abilities', Proc. Natl. Acad. Sci. U.S.A. 79, 2554-2558, 1982.

[4] Psaltis, D., Park, C.H., (1986), 'Nonlinear Discriminant Functions and Associative Memories', J. Denker (Ed), AIP Conference Procc, N. York, Am. Inst. of Phys., p. 370.

\footnotetext{
${ }^{1}$ Partially supported by DTI, U. Chile, FONDECYT 91-1211 (EG) and FONDECYT 0047/90 (MM)

*• Dept. Ingeniería Matemática, U. de Chile, from December 1993
} 